\title{
DÜBLIN
}

Technological University Dublin

ARROW@TU Dublin

\section{A review of Doppler Ultrasound Quality Assurance Protocols and Test Devices}

Jacinta Browne

Technological University Dublin, jacinta.browne@tudublin.ie

Follow this and additional works at: https://arrow.tudublin.ie/scschphyart

Part of the Atomic, Molecular and Optical Physics Commons

\section{Recommended Citation}

Browne, J. : A review of Doppler Ultrasound Quality Assurance Protocols and Test Devices, Physica Medica, 2014. doi:10.1016/j.ejmp.2014.08.003

This Article is brought to you for free and open access by the School of Physics \& Clinical \& Optometric Science at ARROW@TU Dublin. It has been accepted for inclusion in Articles by an authorized administrator of ARROW@TU Dublin. For more information, please contact arrow.admin@tudublin.ie, aisling.coyne@tudublin.ie, gerard.connolly@tudublin.ie.

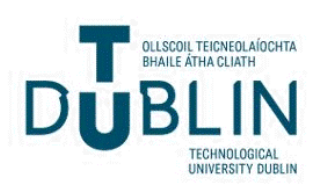


Invited paper

\title{
A review of Doppler ultrasound quality assurance protocols and test devices
}

\author{
Jacinta E. Browne \\ Medical Ultrasound Group, School of Physics and IEO, FOCAS Institute, Dublin Institute of Technology, Kevin Street, Dublin 8, Ireland
}

\section{A R T I C L E I N F O}

\section{Article history:}

Received 19 March 2014

Received in revised form

13 August 2014

Accepted 14 August 2014

Available online 8 September 2014

\section{Keywords:}

Doppler ultrasound quality assurance

Doppler test devices

Flow phantoms

String phantoms and Doppler ultrasound performance

\begin{abstract}
A B S T R A C T
In this paper, an overview of Doppler ultrasound quality assurance (QA) testing will be presented in three sections. The first section will review the different Doppler ultrasound parameters recommended by professional bodies for use in QA protocols. The second section will include an evaluation and critique of the main test devices used to assess Doppler performance, while the final section of this paper will discuss which of the wide range of test devices have been found to be most suitable for inclusion in Doppler QA programmes. Pulsed Wave Spectral Doppler, Colour Doppler Imaging QA test protocols have been recommended over the years by various professional bodies, including the UK's Institute of Physics and Engineering in Medicine (IPEM), the American Institute for Ultrasound in Medicine (AIUM), and the International Electrotechnical Commission (IEC). However, despite the existence of such recommended test protocols, very few commercial or research test devices exist which can measure the full range of both PW Doppler ultrasound and colour Doppler imaging performance parameters, particularly quality control measurements such as: (i) Doppler sensitivity (ii) colour Doppler spatial resolution (iii) colour Doppler temporal resolution (iv) colour Doppler velocity resolution (v) clutter filter performance and (vi) tissue movement artefact suppression. In this review, the merits of the various commercial and research test devices will be considered and a summary of results obtained from published studies which have made use of some of these Doppler test devices, such as the flow, string, rotating and belt phantom, will be presented.
\end{abstract}

() 2014 Associazione Italiana di Fisica Medica. Published by Elsevier Ltd. All rights reserved.

\section{Introduction}

The importance of assessing the quality of Doppler ultrasound systems is apparent considering that the result or product of Doppler ultrasound examinations is frequently directed toward a well-defined clinical question concerning blood flow [1]. For example, Doppler ultrasound is used to determine the level of stenosis by examining the changes in blood flow in the vessel of interest, and to determine whether flow is present in a tight stenosis. Another use is to determine if changes have occurred in blood flow in transplanted kidneys [1-3]. Indeed, the use of Doppler ultrasound has become more widespread over the last decade, largely due to advances in transducer technology, digital electronics and clutter filter algorithms, with a corresponding improvement in the Doppler sensitivity, axial resolution within the sample volume and the low velocity detection capabilities of modern systems [3]. With this increasing use of Doppler ultrasound techniques, it is of

E-mail address: jacinta.browne@dit.ie. paramount importance that ultrasound systems meet the requirements of each of the different clinical applications and that the quality of the information is maintained throughout the lifetime of the ultrasound system. To this end, performance and quality control tests are carried out to determine firstly that the requirements of the clinical examination are achieved at acceptance testing, and then through routine quality assurance testing to ensure that this appropriate level of performance is maintained [4]. Both the absolute performance of Doppler ultrasound and the maintenance of that performance are important [5]. The former is important given that clinicians need to know the accuracy of the maximum velocity detectability of the system, and also to know the system's detection limits in terms of the weakest Doppler signal that can be detected. For example, in the case of the accuracy of the system where the maximum peak systolic velocity is overestimated by $10 \%$ for a patient with an actual peak systolic velocity of $125 \mathrm{~cm} \mathrm{~s}^{-1}$ the measured velocity would be $138 \mathrm{~cm} \mathrm{~s}^{-1}$ which would cause this patient to be miscategorised as a $>50 \%$ stenosis rather than $<50 \%$ stenosis which would possibly alter the treatment regime from drug treatment to surgery [6]. Furthermore, changes in 
Table 1

Overview of the different Doppler parameters for the Doppler modes as recommended by the two professional bodies, IPEM and AIUM.

\begin{tabular}{|c|c|c|c|}
\hline Doppler test parameter & Professional body & Doppler mode ${ }^{a}$ & Doppler test device \\
\hline $\begin{array}{l}\text { 1. Maximum velocity accuracy - This test provides an assessment of the accuracy of the Doppler } \\
\text { system's estimate of the maximum Doppler scatterer velocity. This is one of the most } \\
\text { common measurements made using Doppler ultrasound and provides information } \\
\text { concerning the degree of arterial stenosis, or of the pressure drop across cardiac valves } \\
\text { in a patient. }\end{array}$ & IPEM, IPSM \& AIUM & $\mathrm{S}$ & $\begin{array}{l}\text { String phantom } \\
\text { Flow phantom } \\
\text { Rotating phantom } \\
\text { Rotating torus phantom } \\
\text { Belt phantom } \\
\text { Vibrating disk phantom } \\
\text { Oscillating thin film phantom } \\
\text { Acoustic/electronic } \\
\text { injection test phantom }\end{array}$ \\
\hline $\begin{array}{l}\text { 2. Spectral broadening - The Doppler beam is produced by a group of elements within an array, } \\
\text { known as the active aperture. This results in the Doppler beam insonating the vessel at a } \\
\text { range of angles. Therefore, this leads to a spreading of the range of Doppler shift frequencies }\end{array}$ & IPEM, IPSM & $\mathrm{S}$ & $\begin{array}{l}\text { String phantom } \\
\text { Vibrating point source } \\
\text { phantom }\end{array}$ \\
\hline
\end{tabular}

the blood flow itself.

3. Mean velocity estimation - This test provides an assessment of the accuracy of the Doppler system's estimate of the mean Doppler scatterer velocity. It is also the accuracy of the colour Doppler estimate of the mean scatterer velocity. Quantitative and semi-quantitative analysis of flow in vessels is being used more frequently in the clinical setting, therefore, this requires high accuracy of velocity estimation.

4. Flow direction - this tests the system's ability to distinguish between flow towards and away from the transducer.

5. Axial range gate - is the extent over which the Doppler gate can detect a blood flow signal. IPEM, IPSM

IPEM, IPSM \& AIUM S

6. Sample volume registration - measures the system's range gate sensitivity to check if it is most sensitive at the centre of the gate.

7. Angle correction software accuracy - measures the accuracy of the angle correction software of the system.

8. Highest detectable velocity - Highest detectable velocity is the highest velocity which it is possible to display unambiguously on the PW Doppler spectrum or the colour image. Velocities in the presence of an arterial stenosis or a cardiac valvular narrowing can reach up to $5-6 \mathrm{~m} \mathrm{~s}^{-1}$ and it is desirable for the Doppler mode to display these velocities without aliasing.

9. Lowest detectable velocity - The lowest detectable velocity is the lowest velocity that it is possible to display unambiguously. Visualisation of low velocity flow is necessary in venous flow detection or in tight carotid artery stenosis to distinguish between vessel occlusion and vessel patency.

10. High-pass filter response - This is the ability of the clutter filter to remove strong signals from the vessel wall movement, while still preserving the low velocity content of the flow signal.

11. Penetration depth - This is the maximum depth of a vessel in tissue from which a $50 \mathrm{cms}^{-1}$ Doppler signal free of extraneous noise can be obtained, which represents a typical or average physiological velocity. In clinical practice it is often desirable to obtain signals from major vessels within the body, and also from small vessels for assessment of perfusion.

Sensitivity (Signal-to-Noise ratio)

12. - is the minimum detectable signal level free from extraneous noise. Sensitivity is the most important aspect of Doppler performance, since if flow cannot be detected no other aspect of performance matters.

13. Dynamic range - is defined as the ratio between the maximum clutter signal and the minimum detectable flow signal while both signals are present (the clutter-to-signal ratio). Clinically, it is important to have a good dynamic range particularly for Spectral Doppler when perfusion at the arteriolar level is being measured in solid tissue, for example, in the kidneys, liver or testicles.

14. Spatial Resolution - This is the minimum separation in space for which two separate point or line targets can be resolved or the point spread function of a point source. Visualisation of small areas of flow is required, for example for small vessels, or for regions near to minor degrees of atheroma.

15. Temporal Resolution - This is the minimum separation in time for which two separate events can be identified. Flow events may change very rapidly, particularly for flow in the heart, and a high frame rate is needed to follow these changes.

16. Velocity Resolution - This is the minimum discernible velocity difference of a colour flow image. Quantitative and semi-quantitative analysis of flow in vessels is being used more frequently in the clinical setting, therefore, which necessitates a high accuracy of velocity estimation.

IPSM \& AIUM
IPEM, IPSM \& AIUM S

IPSM \& AIUM $\quad S \& C$

IPSM \& AIUM

$\mathrm{S} \& \mathrm{C}$

IPSM \& AIUM

$S \& C$

IPSM

IPSM \& AIUM

IPSM \& AIUM

$C \& P$

IPSM \& AIUM

C

IPSM

C

Rotating phantom
Acoustic/electronic injection test phantom

String phantom Flow phantom Acoustic grid phantom

Rotating phantom

Rotating torus phantom

String phantom

Flow phantom

Rotating torus phantom

Belt phantom

String phantom

Rotating torus phantom

Belt phantom

Acoustic/electronic

injection test phantom

Flow phantom

Vibrating point

source phantom

String phantom

Flow phantom

source point

String phantom

Flow phantom

String phantom

whan

Rotating torus phantom

phantom

ng phantom

Rotating phantom

Rotating torus phantom

Belt phantom

Acoustic/electronic

injection test phantom

Flow phantom

Rotating phantom Rotating torus phantom 
Table 1 (continued)

\begin{tabular}{|c|c|c|c|}
\hline Doppler test parameter & Professional body & Doppler mode ${ }^{a}$ & Doppler test device \\
\hline $\begin{array}{l}\text { 17. Colour/power Doppler Duplex priority control/Tissue Movement Suppression - This is the } \\
\text { degree of colouring in the tissue region compared to that in a vessel. Colour flow signals may } \\
\text { arise from tissue motion as well as from moving blood. Colour flow signals associated with } \\
\text { tissue motion are generally considered to be undesirable and the ability of the machine to } \\
\text { suppress tissue motion signals is a very important feature. }\end{array}$ & IPSM \& AIUM & $S \& C$ & Belt phantom \\
\hline
\end{tabular}

${ }^{\text {a }} \mathrm{S}=\mathrm{PW}$ spectral Doppler, $\mathrm{C}=$ Colour Doppler Imaging, $\mathrm{P}=$ Power Doppler Imaging.

performance over time due to deterioration is important to quantify, particularly where Doppler ultrasound is used longitudinally to assess changes in a patient's condition over time.

This paper will be divided into three main sections: the first section will provide a review of the current quality assurance protocols which have been recommended by the various international professional organisations; the second section will provide a description and critique of commercial and laboratory test devices currently available for Doppler testing as well as discussing which Doppler parameters have been evaluated using these devices; and the final section will consider what tests should be included in a Doppler quality assurance programme, given the main findings from previous studies investigating the efficacy of different Doppler quality assurances parameters.

\section{Doppler quality assurance protocols}

Doppler Ultrasound quality assurance (QA) protocols have been recommended over many years by several international professional bodies such as the UK Institute of Physics and Engineering in Medicine (IPEM), the American Institute for Ultrasound in Medicine (AIUM) as well as the International Electrotechnical Commission (IEC) who have all devised and recommended both standards for testing and the corresponding test devices which should be used to this end. Each of these Doppler test protocols include test procedures for the evaluation of Pulsed Wave (PW) spectral Doppler, Duplex, Colour and Power Doppler Imaging; these recommended tests can be divided into three main areas of testing: (i) accuracy (ii) sensitivity and (iii) imaging performance.

The identification of appropriate test procedures or test devices to evaluate the performance of Doppler ultrasound systems is difficult as there is no consensus among professional bodies or indeed researchers in this area regarding standard set-up conditions for these tests in terms of ultrasound systems' acquisition parameter settings. Furthermore, there are no recommendations for a standard test device. The lack of such recommendations for both acquisition procedures and test devices presents a difficulty to hospital-based medical physicists in terms of the implementation of a suitable Doppler ultrasound quality assurance programme. A positive step in this direction came from Di Nallo et al. who, in 2006, presented a possible quality control procedure for use with a String Phantom - this will be discussed later in the review in relation to the usefulness of a string phantom test object [7].

The recommended tests by two professional bodies, namely IPEM's report 102 and AIUM's report 2002 are reviewed in this section to extract the main test parameters which should be considered for inclusion in a quality assurance programme for Doppler ultrasound [1,5]. The IPEM produced a new set of recommendations which were outlined in a report published in 2010 ("IPEM Report number 102") [5]; the stated aim of the authors of this report was to establish a specific group of tests which would allow aspects of an ultrasound scanner's performance which were considered likely to deteriorate to be monitored. This report combined all aspects of ultrasound quality assurance and safety testing, and described acceptance, baseline/routine and users' tests which should be carried out, together with recommended time intervals, to ensure the quality of the diagnostic information provided by the ultrasound system. Interestingly, the report only recommended certain tests which evaluate aspects of the accuracy of Doppler ultrasound, largely in recognition of the fact that there is limited availability of commercially-available Doppler test devices and also due to the lack of evidence supporting the wider range of tests which had previously been recommended by the IPSM (the IPEM was formely the Insititute of Physical Sciences in Medicine (IPSM)) in their report published in 1994 [8]. The recommended tests are:

(i) maximum velocity accuracy,

(ii) spectral broadening,

(iii) mean velocity estimation,

(iv) flow direction,

(v) axial range gate,

(vi) Doppler gain control function response,

(vii) pulse repetition frequency (velocity) range control,

(viii) baseline shift,

(ix) angle correction,

(x) colour/power Doppler Duplex priority control, and

(xi) colour/power box positioning and sizing.

The report also includes a description of Doppler ultrasound quality assurance tests which could be included in an expanded test protocol if appropriate resources and expertise was available. The tests included in this section were:

(i) velocity estimation accuracy and velocity resolution of tissue Doppler imaging,

(ii) colour and power Doppler imaging spatial resolution, and

(iii) sensitivity.

Furthermore, the authors indicated that the tests outlined in the earlier IPSM report number 70 were still relevant, but with the caveat that the tests should only be performed if evidence of its ability to monitor changes in performance could be obtained which supported their inclusion in a quality assurance programme [8].

The AIUM report, published in 2002 [1] recommended that Doppler performance be evaluated in terms of:

(i) the detectability of flow,

(ii) Doppler shift frequency (velocity) measurement,

(iii) measurement of derived parameters, and

(iv) colour and power Doppler imaging.

In this report, the authors argued that the detectability of blood flow is the most critical aspect of Doppler performance, since if flow cannot be detected, no other aspect of performance matters. The AIUM's definition of the parameter called "detectability" includes aspects of the quality of the Doppler signal and sources of noise; this parameter is similar to the test parameter "sensitivity" which was recommended as additional test in the IPEM report number 102. The full list of tests recommended by the AIUM report are: 
(i) sensitivity (Signal-to-Noise ratio),

(ii) dynamic range (signal-to-clutter ratio),

(iii) peak velocity accuracy,

(iv) sample volume registration,

(v) angle correction software accuracy,

(vi) directional accuracy,

(vii) high-pass filter response,

(viii) frequency response linearity,

(ix) colour/power Doppler spatial resolution, and

(x) colour/power Doppler Duplex priority control.

There is significant overlap in the test parameters recommended and prioritised by both professional bodies apart from the sensitivity, which was not as strongly recommended by IPEM. However, this can be accounted for if one considers the perspectives taken by each body: the IPEM have recommended tests whose ability to detect changes is supported by an evidence-base and which are also likely to deteriorate over time, whereas the AIUM have taken the perspective of absolute performance being of prime importance in the assurance of quality. In Table 1, a list is presented of all the Doppler parameters for the different Doppler modes which have been recommended by the two professional bodies, together with their recommended test device in each case. These devices are reviewed in Section 2.

\section{Doppler ultrasound test devices}

There are several types of test devices of varying complexity available for assessing Pulse Wave (PW) spectral Doppler as well for colour and power Doppler modes. However, they usually are focused on measuring one particular test parameter such as velocity accuracy, spatial resolution and clutter filter response [2]. The test devices can be divided into two main groups: those which attempt to mimic the properties of tissue, blood vessels and blood itself, including the flow profiles of blood using pump systems, and those which merely seek to present a moving object (usually not even a fluid) to the scanner for assessment with the various Doppler modes.

\section{Flow phantoms}

Flow phantoms are an example of tissue mimicking phantoms and consist of a block of tissue mimicking material (TMM) surrounding a vessel through which blood-mimicking fluid (BMF) is pumped [9-12]. The profile of the flow through the vessels can be steady or pulsatile $[13,14]$. The TMMs used in flow phantoms have the same requirements as the TMMs used in B-mode phantoms, namely a speed of sound of $1540 \mathrm{~m} \mathrm{~s}^{-1}$ and an attenuation coefficient of between 0.5 and $0.7 \mathrm{~dB} \mathrm{~cm}^{-1} \mathrm{MHz}^{-1}$ [15]. The walls of the vessels used in flow phantoms are usually made of c-flex rubber, which is known to cause distortion of the ultrasound wave as it propagates through it [16-18]. To overcome this problem some researchers have used human blood vessels removed during either autopsy or endarterectomy, or alternatively used wall-less vessels [14,19-23]. More recently, the use of vessel mimicking materials has become more prominent [24-26]. Several types of BMF have been developed $[27,28]$, with the one most closely matching real blood developed as part of an European Commission project [11,12]. The Medical Physics Department in St George's Hospital, London, U.K. developed a flow phantom largely based on the International Electrotechnical Comission (IEC) 61685 recommendations [29] which also included extra targets for the assessment of parameters such as Doppler temporal resolution; however, there are limited papers outlining this work despite the routine use of the system for Doppler ultrasound quality control [30].
The above mentioned phantoms tend to have relatively simplistic vessel shapes. More anatomically complex flow phantoms have also been developed for evaluation of new techniques and for training purposes [2]. The research in this area of phantom development has focussed mainly in the production of carotid artery bifurcation and renal artery phantoms, which aim to mimic the complexity found at these locations with respect to both anatomy/ morphology and flow perfusion $[14,21,23,24,26]$. For a more comprehensive evaluation of the different design aspects of flow phantoms, the reader is referred to two reviews by Hoskins $[2,31]$.

There are a number of different flow phantom designs available commercially which typically incorporate a simplistic vessel design and a form of electronic control of the pump system. Table 2 lists flow phantoms which are available from Gammex-RMI, ATS laboratory systems, Dansk Phantoms, and Shelly Medical Imaging Technologies. It should be noted that in order to make these flow phantoms portable, trade-offs in their designs have been implemented, such as:

- a reduction in the vessel inlet length, resulting in a reduced length within which to establish a laminar flow profile within the vessel volume interrogated by the Doppler modes, with the consequent reduction in the range of velocities available with these phantoms

- a single or at best limited range of vessel diameters, limiting the ability to probe the sensitivity and the spatial resolution of the Doppler modes

- depending on the type of pump used in the system, air bubbles tend to be produced as a result of cavitation in the pump head, even at low velocities, which can distort the Doppler spectrum [8].

The Doppler test parameters which have been evaluated using flow phantoms include the following: velocity accuracy; directional discrimination; displayed position of Doppler sample volume; penetration depth; sensitivity; lowest detectable velocity; and spatial resolution [22,27,32-36]. A number of investigators have

Table 2

Commercially available Doppler test devices.

\begin{tabular}{lll}
\hline & Company & Website \\
\hline $\begin{array}{l}\text { Flow phantom } \\
\text { Gammex 1425A LE }\end{array}$ & Gammex RMI & www.gammex.com \\
$\begin{array}{l}\text { Gammex 1430 LE } \\
\text { Model 368 }\end{array}$ & Dansk Fantom & http://www.fantom.dk/ \\
Model 427 & Service & \\
Model 447 & & \\
Model 450 & & \\
Model 453 & & \\
Model 518 & & \\
Agar based & Shelly Medical & www.simutec.com \\
carotid bifurcation & Imaging & \\
flow phantoms & Technologies & \\
(9 models) & & \\
Model U-245 & & \\
Two vessel Doppler & & \\
phantom & & \\
Model 700 Models & ATS Laboratories & www.atslabs.com \\
523 \& 523A Model & & \\
527 Model 750 & & \\
String phantom & & \\
CIRS Model 043 & CIRS Tissue & www.cirsinc.com \\
Doppler String & Simulations & \\
Phantom & \& Phantom & \\
Electronic Injection & Cochnology & \\
NickelTM & UNISYN Medical & http:/www.unisynmedical.com \\
& Technologies & \\
\hline
\end{tabular}




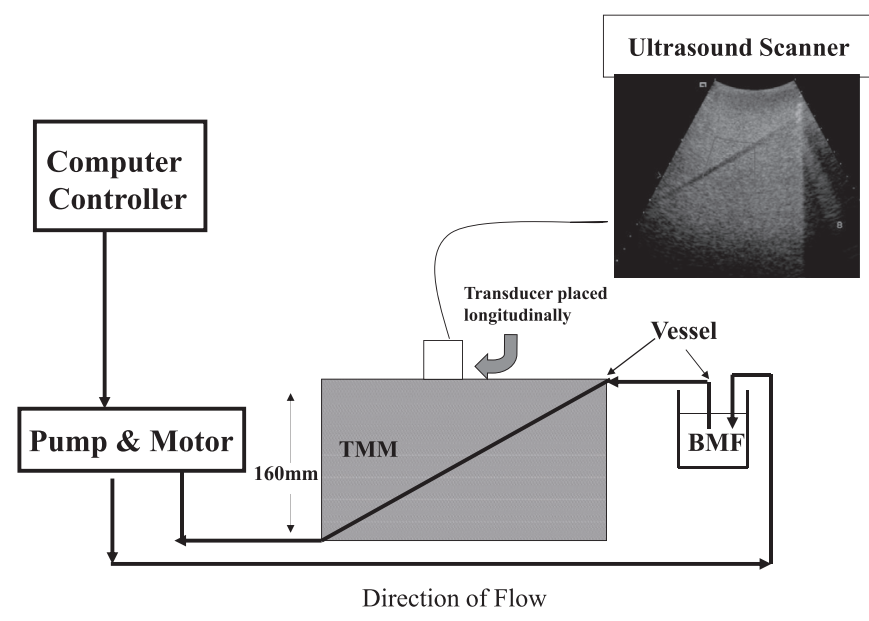

Figure 1. Doppler Sensitivity Flow Phantom set-up and corresponding Doppler Sensitivity Colour Doppler for a curvilinear 2-5 $\mathrm{MHz}$ transducer.

successfully used flow phantoms to investigate the accuracy of the maximum velocity estimation by PW spectral Doppler $[9,10,16,27,37]$. Steinman et al. investigated the sources of error which result in the maximum velocity being overestimated by PW Doppler systems using a flow phantom and found that the effects of Doppler angle and intrinsic spectral broadening were the chief sources of error and concluded that they should be the focus of future efforts to improve the accuracy of the systems [37]. Vachutka et al., used a flow phantom to investigate the effect of dead elements on the accuracy of Doppler ultrasound measurements, maximum and mean velocity as well as the overall power of the Doppler spectrum or colour Doppler signal. In this study a systematic evaluation of the effect of dead elements on the accuracy of Doppler ultrasound measurements was possible as a research ultrasound system Ultrasonix Sonix RP was used to control the number of dead elements through the systematic control of the transducer elements [38]. Furthermore, this study involved the objective analysis of the Doppler ultrasound spectra and colour Doppler images which allowed for subtle changes in performance due to the dead elements to be detected.

Functional tests such as directional discrimination and Doppler sample volume registration have been successfully investigated using a flow phantom since the 1980s [27]. Penetration depth has been investigated very successfully using a flow phantom, given that this device provides an appropriately attenuating medium similar to the in-vivo situation [27]. Browne et al. investigated the use of a custom-built flow phantom to determine the velocity detection limitations of Doppler ultrasound which contained vessels of varying diameters at varying depths (see Fig. 1) [31]. This phantom was designed to produce a stable flow velocity as low as $3 \mathrm{~cm} \mathrm{~s}^{-1}$, making it possible to measure the lowest detectable velocity in vessels of decreasing diameter as a function of penetration depth. From these measurements, the authors were able to define a "Doppler Sensitivity Performance Index" which was found to offer excellent differentiation in the performance levels of ultrasound systems of a similar class, identifying it as a useful Doppler parameter. The computer controller used in this study was also capable of producing pulsatile flow, thereby providing a more physiologically realistic signal.

Li et al. constructed an acoustic grid on a vessel which consisted of line pairs of attenuating material superimposed on a vessel of a flow phantom submerged in water and used this approach to provide a measure of lateral spatial resolution with separation sizes between $0.5 \mathrm{~mm}$ and $10 \mathrm{~mm}$ [35]. However, the arrangement of the acoustic grids may have caused diffraction of the ultrasound beam, which may have affected the colour Doppler spatial resolution measurements. In a separate study, Browne et al. developed two flow phantoms to assess colour Doppler spatial resolution. The first consisted of a series of line pair vessels with fixed separations of $0.6 \mathrm{~mm}, 0.8 \mathrm{~mm}, 1 \mathrm{~mm}$ and $1.2 \mathrm{~mm}$, with the vessels angled at $60^{\circ}$ in order to assess the Doppler performance as a function of depth (Fig. 2a) [33]. While this phantom was able to differentiate the performance of curvilinear transducers between new and older systems, it was not able to adequately challenge linear array transducers, as can be seen in Fig. 2a. In the second phantom, the pairs of vessels diverged at an angle of $1.7^{\circ}$ producing a continuously varying lateral separation ranging between $1 \mathrm{~mm}$ and $1.6 \mathrm{~mm}$ $( \pm 0.2 \mathrm{~mm})$ at a depth of $2 \mathrm{~cm}$. This phantom allowed the minimal resolvable distance to be identified based on imaging a continuous range of separations; however, in practice, some degree of uncertainty in the average separation being probed remained, and furthermore the minimum separation $(1 \mathrm{~mm})$ between the vessels was too large to adequately test the spatial resolution performance of linear array transducers. Colour Doppler images acquired using this phantom are presented in Fig. 2b, demonstrating resolvable signals from 2 vessels of diameter $1.9 \mathrm{~mm}$ separated by $0.6 \mathrm{~mm}$. The parallel line pair phantom, on the other hand, was easier to set up and had a narrower minimum separation of $0.6 \mathrm{~mm}$, but only four discrete separations were available with this phantom [36].

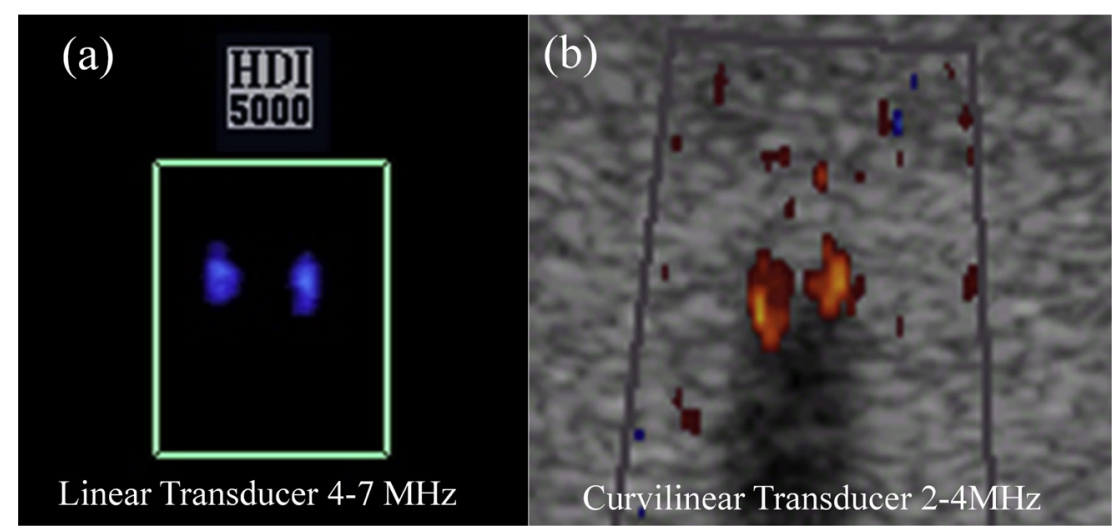

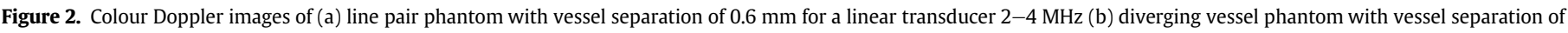
$0.8 \mathrm{~mm}$ for curvilinear $2-5 \mathrm{MHz}$ transducer. 


\section{String phantoms}

Despite the major research interest in the development of flow phantoms, a number of non-tissue mimicking Doppler test objects have been developed. The most common test object which has been discussed in the literature is the string phantom, which consists of a filament attached to a series of pulley wheels and a drive wheel contained within a water tank [39-46]. The drive wheel is driven by a motor, which can be controlled directly by an electronic controller or via a computer, to produce steady or pulsatile movement of the filament. The tissue medium used in these test devices is usually water or speed of sound corrected water, which does not offer an attenuation similar to that which occurs in-vivo [40]. Hoskins carried out an investigation into the effect of the filament material on the Doppler spectrum and found that the backscatter from the spiral wound filaments which are typically used (such as silk) changed over time due to the gradual release of trapped air from the filament [41]. It was also found that the backscatter power changed as a function of angle due to the spiral pattern, producing a Bragg-like scattering. Hoskins recommended the use of O-ring rubber as a suitable filament for use with the string phantom; however, the O-ring rubber produces a strong specular refection which is not representative of typical signals from blood [42]. As a result, when this type of filament is used with a string phantom, the acoustic output and Doppler gain setting must be reduced in order to prevent saturation in the Doppler spectrum [5].

Only one string phantom is currently available commercially, the Model 043 from CIRS Tissue Simulations \& Phantom Technology, USA (www.cirsinc.com); details are provided in Table 2. However, it should be noted that this phantom has several limitations, including: (1) the use of a silk filament as the Doppler target; (2) the fact that the filament passes through an air-water interface, resulting in air bubbles becoming trapped on the filament; and (3) the motor produces strong vibrations at certain velocities which effects the Doppler measurement accuracy at these specific velocities. Cournane et al. investigated the use of this string phantom as part of a routine quality assurance programme and made recommendations to overcome some of the limitations associated with the design [44]. A string phantom which was previously available commercially is the DP1 (BBS Medical Electronic AB, Hagersten, Sweden) and this was used in a large number of the aforementioned studies which investigated the effect of filament material on the Doppler spectrum characteristics $[41,42,45,46]$. This string phantom design incorporated a submergible motor and so the filament remained in the fluid throughout the test cycle and so it did not have the issue of dragging air bubbles along the filament at an air/water interface.
The Doppler test parameters which have been evaluated using string phantoms are: maximum velocity accuracy, spectral broadening, lowest and highest detectable velocities, penetration depth, direction detection accuracy, sensitivity $[7,39,44]$. A representative Doppler spectrum of the HDI3000 curvilinear 2-4 MHz transducer using an in-house string phantom based on the BBS string phantom design is presented in Fig. 3 [45]. This test device is ideal for measuring the maximum velocity accuracy and the intrinsic spectral broadening, given that it presents a single velocity source to the ultrasound scanner [6]. Hoskins assessed the maximum velocity accuracy of six different ultrasound scanners using the BBS string phantom which enabled a direct comparison to be made between the Doppler-estimated maximum velocity and the true filament velocity. It was found that under standard patient set-up conditions, the maximum velocity was overestimated in all cases, with errors of up to $29 \%$. Such large errors could result in some patients being incorrectly categorized, considering that errors in maximum velocity accuracy would be directly translated into corresponding errors in velocity-derived estimates of vessel stenosis. Walker et al. investigated the maximum velocity accuracy of three cardiac systems using the BBS string phantom and found that one ultrasound system consistently overestimated velocity by about $5 \%$, whereas the other two systems were quite accurate in velocity estimation [46]. This provided evidence of the importance of acceptance testing ultrasound systems, particularly in relation to the maximum velocity accuracy. In addition to maximum velocity accuracy, string phantoms have also been recommended by a number of investigators as a useful test device for other Doppler test parameters such as spectral broadening, lowest and highest detectable velocities, penetration depth, direction detection accuracy, range gate registration and sensitivity $[7,39,43,44,47,48]$. In 2006, Di Nallo et al. presented a possible quality control procedure for use with a String Phantom. This protocol included the evaluation of Doppler penetration depth, sensitivity, sample volume dimensions, Doppler angle, peak velocity accuracy, colourised noise, colour contrast, temporal and spatial resolution, aliasing and sensitivity performance index through the use of objective analysis of the Doppler data [7]. In this study faults were detected at baseline in terms of the functionality of the ultrasound systems, and the authors indicated that a longitudinal study evaluating the efficacy of this protocol was underway. However, unless an attenuating medium such as a rubber block is placed in front of the transducer or castor oil is added to the fluid surrounding the string, accurate estimates of penetration depth and sensitivity cannot be obtained using a string phantom as appropriate attenuation is not produced $[39,43]$.

Lange and Loupas evaluated the spatial resolution of colour Doppler using a modified string phantom, which consisted of two
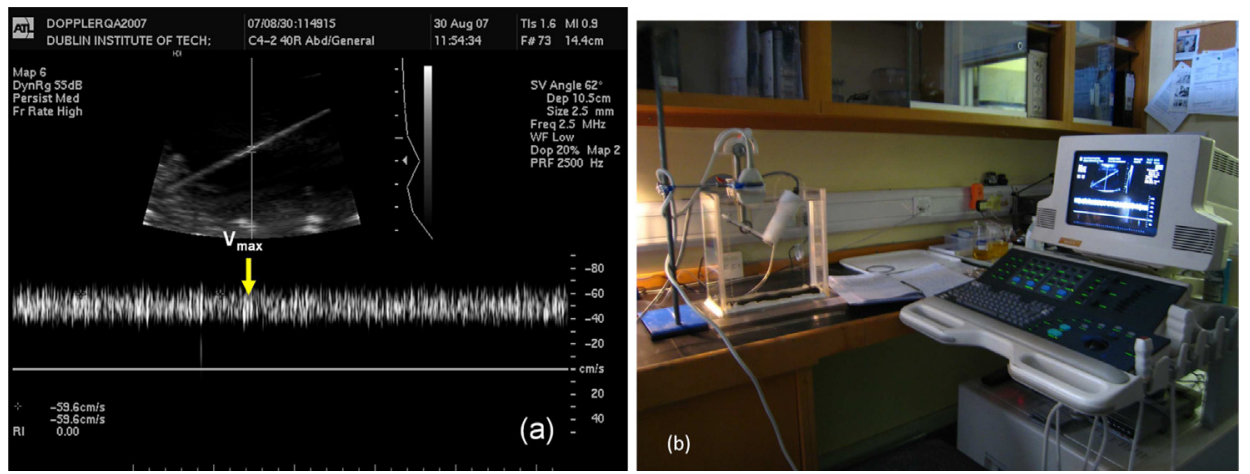

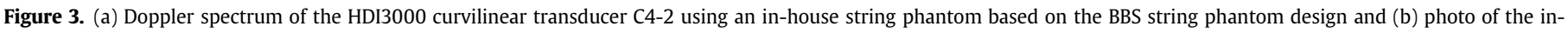
house string phantom based on the BBS design. 
filaments with an adjustable spacing which facilitated the assessment of spatial resolution [49]. This approach provided a measure of spatial resolution with separation sizes between $0.5 \mathrm{~mm}$ and $10 \mathrm{~mm}$. However, the measurements were carried out using O-ring rubber, which has a significantly higher backscatter value than blood, and to further compound this problem the measurements were carried out in water. Therefore, the resulting colour Doppler signal was significantly stronger than typical blood signals. Furthermore, string phantoms are not particularly suitable for measuring colour Doppler spatial resolution because, instead of producing a volume of flow, they produce a narrow line of flow.

\section{Other Doppler test devices}

A range of other Doppler test devices have been developed in research laboratories over the years, such as: the rotating phantom, rotating torus phantom, belt phantom, vibrating disk, oscillating thin film test object and electronic injection devices, each of which will be discussed in turn in this section.

In order to produce a range of velocities as occurs in a blood vessel in-vivo, McDicken et al. constructed a test device consisting of a circular disk of reticulated foam immersed in water which could be rotated around a central axis [50]. In order to improve the tissue equivalence of the rotating phantom, in a follow-on study the investigators successfully produced a rotating phantom with blocks of tissue mimicking material in place of the reticulated foam, which facilitated the investigation of the performance of tissue Doppler as a source of moving tissue was used [51]. Fleming et al. developed a range of rotating phantoms and carried out an investigation into the velocity resolution, spatial resolution and accuracy of Doppler images as part of a study into the Doppler display of cardiac tissue motion [51]. One of the developed phantoms consisted of two blocks of gel, one with square grooves cut into it, the other with square ridges. The two pieces of gel could interlock and slide against each other. The blocks were made to oscillate back and forth against each other. The smallest spatial detail that was observed in the Doppler image was $3 \mathrm{~mm}$ by $3 \mathrm{~mm}$. While interesting, this approach to measuring spatial resolution was nonetheless somewhat limited, as it involved subjectively deciding whether the different sized grooves were visible in the Doppler Tissue images, and furthermore could not take into account any differences between axial and lateral resolution.

Another variant on the rotating phantom consisted of a rotating cylinder of TMM with a sector-shaped cut-out throughout its length [52]. This phantom was capable of assessing axial and lateral resolution.

A small-scale rotating phantom made from TMM was developed to assess PW spectral Doppler velocity accuracy of a preclinical ultrasound system (Vevo 770, Visualsonics, Canada) [53]. This design of phantom overcame the problem of realistically evaluating the Doppler performance of very high frequency Doppler as developing a flow phantom with appropriately sized vessels of micron dimensions would be technically very challenging. It was found that the maximum velocity was overestimated by up to $158 \%$ by spectral Doppler on this system. The results from this phantom were compared with the results of a ray model of geometric spectral broadening used to predict velocity errors. It was found that there was good agreement $(<10 \%)$ between the theoretical velocity errors (determined using the ray model of geometric spectral broadening) and measured errors for beam-target angles of $50^{\circ}-80^{\circ}$. However, for angles of $10^{\circ}-40^{\circ}$, the agreement was not as good ( $>50 \%$ ).

Rickey et al. developed a belt phantom which consisted of a layer of reticulated foam stitched onto a rubber belt. It could provide a 2-D representation of flow in terms of a range of velocities and was used to determine the mean velocity accuracy of colour
Doppler [54]. A modified version of the belt phantom, consisting of an acoustic beam splitter which allowed simulated flow and simulated clutter to be interrogated by the transducer simultaneously, was used in a later study to evaluate the performance of colour clutter filters [55]. The majority of the ultrasound systems tested had a lowest detectable velocity of $3 \mathrm{~cm} \mathrm{~s}^{-1}$ or less. The lowest clutter filter setting will strongly influence the lowest detectable velocity which can be determined by an ultrasound system. Therefore, another approach to assessing lowest detectable velocity is through the assessment of the clutter filter performance using a test device such as the modified belt phantom. However, due to the complexity of these belt phantoms, the design has not been replicated elsewhere and consequently there has been a limited amount of research conducted using it.

A rotating torus phantom was a research test object developed by Stewart to evaluate mean velocity accuracy of colour Doppler [56]. Colour Doppler velocity accuracy does not require tissue equivalence of the test object, although it is very important to have an accurate and precisely controlled velocity source which was possible using the rotating torus phantom. A further investigation using this phantom was carried out to assess the effect of instrument settings such as Doppler angle, wall filter setting and pulse repetition frequency on the measurement of the colour Doppler accuracy [57]. Although reproducibility is often more important than absolute accuracy in serial clinical examinations, differences in colour Doppler images due to variations in instrument settings may, nevertheless, be interpreted as having substantial diagnostic meaning [57].

Simple point source vibrating target phantoms have been used to evaluate the sample volume dimensions (i.e. the spatial resolution) of PW spectral Doppler [24,58]. The vibrating point source consisted of a $280 \mu \mathrm{m}$ metal bead attached to a small speaker. The speaker was driven at approximately $72 \mathrm{~Hz}$ with sufficient amplitude to vibrate the small bead within a water bath.

Another vibrating target consisted of a diffusely scattering circular plate, $15 \mathrm{~cm}$ in diameter [59]. Using this vibrating disk, it was possible to precisely control the frequency output and the amplitude of the signal produced. Wang et al. used this vibrating disk phantom to evaluate colour Doppler sensitivity, as it was possible to precisely control the signal amplitude. However, due to the bidirectional symmetrical side bands, ambiguity was produced for colour Doppler systems and, consequently, reliable measurements of colour/power Doppler sensitivity could not be made using this test object.

Phillips et al. developed an oscillating thin film test object, consisting of a number of precisely deposited sub-resolvable scatters for making spatial resolution and sensitivity measurements in colour Doppler $[60,61]$. The thin film consisted of a thin planar substrate that was acoustically matched to the surrounding media. The scatterers were precisely located and patterned on the surface of the substrate which ensured the production of highly reproducible test object with controllable scattering characteristics. It was possible to produce a time-varying flow velocity which simulated an arterial flow pattern, including its characteristic velocity distribution in forward and reverse directions simultaneously. This test device produced a bi-directional flow which meant that the Doppler shift signal produced by this test device had equal contributions in the positive and negative sideband resulting in a spectral Doppler trace with a positive and negative Doppler signal.

Another type of Doppler test object is an electronic injection device. This device has no moving parts, but instead artificially synthesises the Doppler signal electronically and injects it into the Doppler measurement system in the ultrasound scanner. There are two approaches used in electronic injection of the Doppler signal: direct injection, in which the signal is directly injected at 
some point in the signal processing chain, and acoustic injection, in which the acoustic signal is produced by a separate transducer which is then detected by the Doppler instrument under test [62-66]. The most recent of these electronic injection test devices is the Leicester Electronic Doppler Phantom (Medical Physics Department, Leicester Royal Infirmary, UK) which uses modern digital signal processing methods and field programmable gate array technology to overcome some of the limitations of previously described electronic phantoms [66]. Using this device, it is possible to evaluate frequency response linearity and range gate characteristics, dynamic range and velocity measurement accuracy. The use of direct acoustic coupling eliminates uncertainties caused by Doppler beam effects, such as intrinsic spectral broadening, but as a result prevents the evaluation of intrinsic spectral broadening.

There is currently one acoustic test device available commercially, the Nickel' ${ }^{\mathrm{TM}}$ (Unisyn, USA - Table 2); this compact handheld acoustic injection test object senses the acoustic pulses emitted by the elements of the ultrasound transducer under test, and then generates an acoustic signal simulating a target which it transmits back towards and is detected by the transducer under test. The device can be used to assess the functionality of the PW spectral Doppler (by producing a velocity between 50 and $80 \mathrm{~cm} \mathrm{~s}^{-1}$ depending on the Doppler shift resulting from transmit frequency of the system under test. Furthermore, it produces a line or block of colour to assess the quality of the colour Doppler signal. There have been no studies to date reporting the use of this commercial test object in routine quality assurance programmes.

Electronic and acoustic injection research test objects have been used to measure colour Doppler directional accuracy, high-pass filter response and frequency response linearity [62-66].

\section{Discussion}

The professional bodies, IPEM and AIUM have recommended a range of Doppler test parameters for inclusion in Doppler quality assurance programmes $[1,5]$. The IPEM report recommends test parameters which primarily evaluate the accuracy of Doppler measurements and provides a measure of acceptance for the system in terms of pass or fail. The main reasons for this approach being recommended in the IPEM report is because there is currently a lack of evidence regarding what routine tests can provide information about changes in the Doppler performance over time. However, it is suggested that those involved in Doppler ultrasound quality assurance should gather evidence for example as part of reactive testing when a fault is detected as a means to gathering evidence for the tests efficacy in faulty detection. The AIUM report, on the other hand, recommends test parameters which evaluate all aspects of Doppler performance without reference to the availability of commercial test devices and with no reference to the requirement for evidence as to the suitability of the test, in terms of detecting changes over time. In the IPEM's supplemental testing section, further tests are described for the evaluation of the detection limits and resolution of Doppler ultrasound if time and resources were available, although it was recommended that the clinical function of the specific Doppler ultrasound system under test should be considered in order to develop an appropriate quality assurance programme tailored to its usage [5]. Given the lack of evidence regarding the efficacy of the different Doppler quality control tests the more sensible approach seems to be that which is recommended in the IPEM report 102 [5]. That is to carry out the main acceptance and functionality tests as well as gathering evidence regarding the efficacy of different routine tests as obtained through reactive testing.
Of the main Doppler quality control tests maximum velocity accuracy has been found to be sensitive to element drop-out in a number of studies $[38,44]$. However, the question remains would the paper clip test provide this information before changes in maximum velocity accuracy have occurred. A number of investigations have been carried out to determine the answer to this question for routine B-mode QC testing, where a comparison between B-mode phantom measurements, visual assessment and test for element degradation or drop-out was made and found that this paper clip test, for element degradation or drop-out, detected more faults [67-70]. There is currently insufficient longitudinal data which can provide evidence regarding the efficacy of the different Doppler quality assurance parameters and so there is a requirement for the medical physics community to collect data which will inform the decision of which tests should be included in a Doppler ultrasound quality assurance programme.

The majority of test devices described in this review are not commercially available, and those which are commercially available are not capable of evaluating all aspects of PW spectral and colour Doppler performance as part of a quality assurance programme; this is part of the reason that professional bodies are reluctant to recommend any one particular Doppler test device for routine quality assurance. Furthermore, the absence of a single test device capable of testing all aspects of Doppler performance is another reason for the limited implementation of Doppler quality assurance programmes in the hospital environment. Consequently, there is a need for a Doppler test device which will reliably and accurately determine the full range of PW spectral and colour Doppler test parameters. In the absence of such an ideal Doppler test device, a decision has to be made regarding what test device one should chose for a Doppler quality assurance programme. The first question which should be considered is the specific clinical function of Doppler ultrasound measurements to which a particular machine is being used, which in turn can help identify the most important aspect of performance to measure. This may be the measurement of maximum velocity, or perhaps the detection of the presence of flow, or even the detection of very weak signals (weak due to vessel size or depth from which the signal originates). Once this is clarified, the most appropriate test device can be selected. The information presented in Table 1 outlines the different Doppler test parameters that they assess together with the clinical implication of the performance of this parameter. Reference to suitable phantom design types for measuring this aspect of the Doppler performance is also provided.

Where the measurement of maximum velocity is identified as the most pertinent aspect to assess, the estimation of maximum velocity accuracy would be of utmost importance. Furthermore, it would be important to assess the percentage intrinsic spectral broadening as this will impact the maximum velocity accuracy [37]. Considering the availability of commercial devices, both flow and string phantoms could be used to measure maximum velocity accuracy, although flow phantoms could not be used to measure the percentage intrinsic spectral broadening. Furthermore, either of these test devices could be used to asses other aspects of Doppler performance such as: mean velocity estimation; range gate accuracy; sample volume dimensions; angle correction; highest and lowest detectable velocity and flow direction discrimination.

However, if the detection of the presence of flow or the detection of very weak signals were required, then an estimation of the parameters lowest detectable velocity, sensitivity and penetration depth, would be of utmost importance. In this case, both flow and string phantoms could be used to measure lowest detectable velocity. However, only a phantom which produced attenuation typical of that found in vivo would be suitable for measurements of the sensitivity and penetration depth, hence either a flow phantom 
or a string phantom with an attenuating medium such as castor oil or a liquid TMM, could be used [39,43,71]. For sensitivity and penetration depth performance, the most important factor to be considered in terms of the design of the test device is the degree of tissue equivalence of the test phantom, and flow phantoms tend to be the most tissue equivalent test devices commercially available.

There is a clear need for a test device to be developed and made available commercially that is capable of testing all aspects of PW spectral and colour Doppler imaging. If the design of a single device covering all Doppler aspects proves impossible, an alternative strategy might be to use a set of test devices, such as a string phantom with an easy to implement modification for the evaluation of spatial resolution, as developed by Lange and Loupas [49], and a flow phantom with vessels of small diameters for use in evaluating sensitivity [49]. A key criterion for such an approach, particularly if it were to be adopted into routine practice in busy hospital environments, would be ease of set-up and use to assess the accuracy, detectability and resolution of Doppler ultrasound systems.

\section{Conclusion}

In a busy hospital environment so many clinical decisions are based on Doppler parameter measurements that it is important to undertake regular and accurate QA testing appropriate to the clinical application. In order to make this viable it is important that an easy to set-up test device is used for quality control of Doppler ultrasound and that this test device ensures repeatable and reliable measurements of Doppler performance so that subtle changes in performance over time can be detected and monitored. Objective analysis of the Doppler signal should also be considered so that subtle changes in performance or accuracy can be reliably detected.

\section{References}

[1] AIUM. Performance criteria and measurements for Doppler ultrasound devices. United States of America: American Institute of Ultrasound in Medicine; 2002.

[2] Hoskins PR. Simulation and validation of arterial ultrasound imaging and blood flow. Ultrasound Med Biol 2008;34:693-717.

[3] Evans DH, Jensen JA, Nielsen MB. Ultrasonic colour Doppler imaging. Interface Focus 2011;1:490-502.

[4] Russell S. Ultrasound quality assurance and equipment governance. Ultrasound 2014;22:66-9.

[5] IPEM. Report number 102-quality assurance of ultrasound imaging systems. York, United Kingdom: Institute of Physics and Engineering in Medicine; 2010.

[6] Hoskins PR. Accuracy of maximum velocity estimates made using Doppler ultrasound systems. Br J Radiol 1996;69:172-7.

[7] Di Nallo AM, Strigari L, Benassi M. A possible quality control protocol for Doppler ultrasound for organizational time optimization. J Exp Clin cancer Res: CR 2006;25:373-81.

[8] IPSM Report No 70. Testing of Doppler ultrasound equipment; 1994.

[9] Hoskins PR, Anderson T, McDicken WN. A computer controlled flow phantom for generation of physiological Doppler waveforms. Phys Med Biol 1989;34:1709-17.

[10] Law YF, Cobbold RS, Johnston KW, Bascom PA. Computer-controlled pulsatile pump system for physiological flow simulation. Med Biol Eng Comput 1987;25:590-5.

[11] Ramnarine KV, Nassiri DK, Hoskins PR, Lubbers J. Validation of a new bloodmimicking fluid for use in Doppler flow test objects. Ultrasound Med Biol 1998;24:451-9.

[12] Ramnarine KV, Hoskins PR, Routh HF, Davidson F. Doppler backscatter properties of a blood-mimicking fluid for Doppler performance assessment. Ultrasound Med Biol 1999;25:105-10.

[13] Shortland AP, Cochrane T. Doppler spectral waveform generation in vitro: an aid to diagnosis of vascular disease. Ultrasound Med Biol 1989;15:737-48.

[14] Meagher S, Poepping TL, Ramnarine KV, Black RA, Hoskins PR. Anatomical flow phantoms of the nonplanar carotid bifurcation, part II: experimental validation with Doppler ultrasound. Ultrasound Med Biol 2007:33:303-10.

[15] Browne JE, Ramnarine KV, Watson AJ, Hoskins PR. Assessment of the acoustic properties of common tissue-mimicking test phantoms. Ultrasound Med Biol 2003;29:1053-60.

[16] Law YF, Johnston KW, Routh HF, Cobbold RS. On the design and evaluation of a steady flow model for Doppler ultrasound studies. Ultrasound Med Biol 1989;15:505-16.
[17] Thompson RS, Aldis GK. Effect of a cylindrical refracting interface on ultrasound intensity and the CW Doppler spectrum. IEEE Trans Bio-med Eng 1996;43:451-9.

[18] Steel R, Fish PJ. Lumen pressure within obliquely insonated absorbent solid cylindrical shells with application to Doppler flow phantoms. IEEE Trans Ultrason Ferroelectr Freq Control 2002;49:271-80.

[19] Dabrowski W, Dunmore-Buyze J, Rankin RN, Holdsworth DW, Fenster A A real vessel phantom for imaging experimentation. Med Phys 1997;24: 687-93.

[20] Dabrowski W, Dunmore-Buyze J, Cardinal HN, Fenster A. A real vessel phantom for flow imaging: 3-D Doppler ultrasound of steady flow. Ultrasound Med Biol 2001;27:135-41.

[21] Ramnarine KV, Anderson T, Hoskins PR. Construction and geometric stability of physiological flow rate wall-less stenosis phantoms. Ultrasound Med Biol 2001;27:245-50.

[22] Rickey DW, Picot PA, Christopher DA, Fenster A. A wall-less vessel phantom for Doppler ultrasound studies. Ultrasound Med Biol 1995;21:1163-76.

[23] King DM, Ring M, Moran CM, Browne JE. Development of a range of anatomically realistic renal artery flow phantoms. Ultrasound Med Biol 2010;36:1135-44.

[24] Poepping TL, Nikolov HN, Rankin RN, Lee M, Holdsworth DW. An in vitro system for Doppler ultrasound flow studies in the stenosed carotid artery bifurcation. Ultrasound Med Biol 2002:28:495-506.

[25] King DM, Moran CM, McNamara JD, Fagan AJ, Browne JE. Development of a vessel-mimicking material for use in anatomically realistic Doppler flow phantoms. Ultrasound Med Biol 2011;37:813-26.

[26] King DM, Fagan AJ, Moran CM, Browne JE. Comparative imaging study in ultrasound, MRI, CT, and DSA using a multimodality renal artery phantom. Med Phys 2011;38:565-73.

[27] Boote EJ, Zagzebski JA. Performance tests of Doppler ultrasound equipment with a tissue and blood-mimicking phantom. J Ultrasound Med: Off J Am Inst Ultrasound Med 1988;7:137-47.

[28] Oates CP. Towards an ideal blood analogue for Doppler ultrasound phantoms. Phys Med Biol 1991;36:1433-42.

[29] Comission IE. International electrotechnical comission 61685. Ultrasonicsflow measurement systems-flow object, 2001 test object. Geneva Switzerland; 2001.

[30] Sinha A, Cane C, Kempley S. Blood flow in the common carotid artery in term and preterm infants: reproducibility and relation to cardiac output. Arch Dis Child Fetal Neonatal Ed 2006;91:F31-5.

[31] Hoskins PR. Physical properties of tissues relevant to arterial ultrasound imaging and blood velocity measurement. Ultrasound Med Biol 2007;33: $1527-39$.

[32] Hoskins PR, Li SF, McDicken WN. Velocity estimation using duplex scanners. Ultrasound Med Biol 1991;17:195-9.

[33] Teirlinck CJ, Bezemer RA, Kollmann C, Lubbers J, Hoskins PR, Ramnarine KV, et al. Development of an example flow test object and comparison of five of these test objects, constructed in various laboratories. Ultrasonics 1998;36: $653-60$.

[34] Browne JE, Watson AJ, Hoskins PR, Elliott AT. Validation of a sensitivity performance index test protocol and evaluation of colour Doppler sensitivity for a range of ultrasound scanners. Ultrasound Med Biol 2004;30:1475-83.

[35] Li S, Hoskins PR, McDicken WN. Rapid measurement of the spatial resolution of colour flow scanners. Ultrasound Med Biol 1997;23:591-6.

[36] Browne JE, Brown I, Hoskins PR, Watson AJ, Elliott AT. Colour Doppler spatial resolution performance testing. Ultrasound 2007;15:162-6.

[37] Steinman AH, Tavakkoli J, Myers Jr JG, Cobbold RS, Johnston KW. Sources of error in maximum velocity estimation using linear phased-array Doppler systems with steady flow. Ultrasound Med Biol 2001;27:655-64.

[38] Vachutka J, Dolezal L, Kollmann C, Klein J. The effect of dead elements on the accuracy of Doppler ultrasound measurements. Ultrason Imaging 2014;36 $18-34$.

[39] Goldstein A. Performance tests of Doppler ultrasound equipment with a string phantom. J Ultrasound Med: Off J Am Inst Ultrasound Med 1991;10:125-39.

[40] Goldstein A. Effect of tank liquid acoustic velocity on Doppler string phantom measurements. J Ultrasound Med: Off J Am Inst Ultrasound Med 1991;10: $141-8$.

[41] Hoskins PR. Choice of moving target for a string phantom: I. Measurement of filament backscatter characteristics. Ultrasound Med Biol 1994:20:773-80.

[42] Hoskins PR. Choice of moving target for a string phantom: II. On the performance testing of Doppler ultrasound systems. Ultrasound Med Biol 1994;20: $781-9$.

[43] Russell SV, McHugh D, Moreman BR. A programmable Doppler string test object. Phys Med Biol 1993;38:1623-30.

[44] Cournane S, Fagan AJ, Browne JE. An audit of a hospital-based Doppler ultrasound quality control protocol using a commercial string Doppler phantom. Phys Med 2014;30:380-4.

[45] Walker AR, Phillips DJ, Powers JE. Evaluating Doppler devices using a moving string test target. J Clin Ultrasound 1982;10:25-30.

[46] Walker A, Olsson E, Wranne B, Ringqvist I, Ask P. Accuracy of spectral Doppler flow and tissue velocity measurements in ultrasound systems. Ultrasound Med Biol 2004;30:127-32.

[47] Eicke BM, Kremkau FW, Hinson H, Tegeler CH. Peak velocity overestimation and linear-array spectral Doppler. J Neuroimaging: Off J Am Soc Neuroimaging 1995;5:115-21. 
[48] Eicke BM, Tegeler CH, Howard G, Bennett JB, Myers LG, Meads D. In vitro validation of color velocity imaging and spectral Doppler for velocity determination. J Neuroimaging: Off J Am Soc Neuroimaging 1993;3:89-92.

[49] Lange GJ, Loupas T. Spectral and color Doppler sonographic applications of a new test object with adjustable moving target spacing. J Ultrasound Med: Off J Am Inst Ultrasound Med 1996;15:775-84.

[50] McDicken WN, Morrison DC, Smith DS. A moving tissue-equivalent phantom for ultrasonic real-time scanning and Doppler techniques. Ultrasound Med Biol 1983:9:L455-9.

[51] Fleming AD, McDicken WN, Sutherland GR, Hoskins PR. Assessment of colour Doppler tissue imaging using test-phantoms. Ultrasound Med Biol 1994;20: 937-51.

[52] Murray I, Anderson T, McDicken W. A new test phantom for the evaluation of Doppler tissue images. Ultrasound 2004;12:121-4.

[53] Yang X, Sun C, Anderson T, Moran CM, Hadoke PWF, Gray GA, et al. Assessment of spectral Doppler in preclinical ultrasound using a small-size rotating phantom. Ultrasound Med Biol 2013;39:1491-9.

[54] Rickey DW, Rankin R, Fenster A. A velocity evaluation phantom for colour and pulsed Doppler instruments. Ultrasound Med Biol 1992;18:479-94.

[55] Rickey DW, Fenster A. A Doppler ultrasound clutter phantom. Ultrasound Med Biol 1996;22:747-66.

[56] Stewart SF. A rotating torus phantom for assessing color Doppler accuracy Ultrasound Med Biol 1999:25:1251-64.

[57] Stewart SF. Effects of transducer, velocity, Doppler angle, and instrument settings on the accuracy of color Doppler ultrasound. Ultrasound Med Biol 2001;27:551-64.

[58] Hoeks AP, Ruissen CJ, Hick P, Reneman RS. Methods to evaluate the sample volume of pulsed Doppler systems. Ultrasound Med Biol 1984;10:427-34.

[59] Wang KYBS, Hossack JM. A tool for evaluating Doppler sensivitivity. J Vasc Technol 1992:16:87-94.
[60] Phillips DMS, Parker K. Colour Doppler spatial resolution measurements with an oscillating thin film test object. IEEE Ultrason Symp 1997:1517-20.

[61] McAleavey S, Hah Z, Parker K. A thin film phantom for blood flow simulation and Doppler test. IEEE Trans Ultrason Ferroelectr Freq Control 2001;48: 737-42.

[62] Bastos CA, Fish PJ. A Doppler signal simulator. Clinical physics and physiological measurement : an official journal of the Hospital Physicists' Association. Deutsche Gesellschaft fur Medizinische Physik Eur Fed Organ Med Phys 1991:12:177-83.

[63] Evans JA, Price R, Luhana F. A novel testing device for Doppler ultrasound equipment. Phys Med Biol 1989;34:1701.

[64] Lunt MJ, Anderson R. Measurement of Doppler gate length using signal reinjection. Phys Med Biol 1993;38:1631.

[65] Li SF, Hoskins PR, Anderson T, McDicken WN. An acoustic injection test object for colour flow imaging systems. Ultrasound Med Biol 1998;24:161-4.

[66] Gittins J, Martin K. The leicester Doppler phantom-a digital electronic phantom for ultrasound pulsed Doppler system testing. Ultrasound Med Biol 2010;36:647-55.

[67] Mårtensson M, Bjällmark A, Brodin L-Å. Evaluation of tissue Doppler-based velocity and deformation imaging: a phantom study of ultrasound systems. Eur J Echocardiogr 2011;12:467-76.

[68] Mårtensson M, Olsson M, Brodin L-A. Ultrasound transducer function: annual testing is not sufficient. Eur J Echocardiogr 2010;11:801-5.

[69] Mårtensson M, Olsson M, Segall B, Fraser AG, Winter R, Brodin L-Å. High incidence of defective ultrasound transducers in use in routine clinical practice. Eur J Echocardiogr 2009;10:389-94.

[70] Sipilä O, Mannila V, Vartiainen E. Quality assurance in diagnostic ultrasound. Eur J Radiol;80:519-25.

[71] Madsen EL, Frank GR, Dong F. Liquid or solid ultrasonically tissue-mimicking materials with very low scatter. Ultrasound Med Biol 1998;24:535-42. 